\title{
Chebyshev Series Representation For Product Of Chebyshev Polynomials And Some Notable Functions
}

\author{
Olagunju A. S. \\ (Department of Mathematics and Statistical Sciences, Kwara state University, Malete,Nigeria)
}

\begin{abstract}
In this paper, the challenging difficulties encountered in solving non-polynomial variable coefficients differential equations by the use ofChebyshev expansion method is resolved. In such problems, where $f(x)$ is non-polynomial, there exists the need to express products like $f(x) T_{r}(x)$ in series of Chebyshev polynomials for easy comparison of both sides of the differential equation. Numerical experiments is carried out on notable functions and the results are presented.
\end{abstract}

Keyword:Chebyshev polynomials, taylor's series expansion, non-polynomial variable coefficients.

\section{INTRODUCTION}

One of the most common problems in numerical analysis is the computation of a function $\mathrm{f}(\mathrm{x})$ for one or more given values of its argument $x$. The function may be defined in a variety of ways, either explicitly, as a series, a definite integral or some other computable form; or implicitly for example as the solution of a differential or integral equation ([1], [2],[3] and [4]). In addition, the function may as well occur in its products with other functions say $T(x)$, and in such cases we are confronted with the challenge of expressing these products in computable form.

A vivid illustration is found in the work ofFox [3] where it is apparently noticed that Chebyshev expansion method for non-polynomial variable coefficients fails because of the inability to express variable coefficient problems in its Chebyshev series equivalence before the process of coefficients comparison. For polynomial variable coefficients differential equations, a standard technique is given in [2] where the use of the Chebyshev recurrence relation $T_{n+1}(x)=2 x T_{n}(x)-T_{n-1}(x)$ for expressing such products in Chebyshev form is thoroughly expatiated.

Many of the publications on the use of Chebyshev expansion method for differential equations centres on this polynomial variable coefficients problem and those that ventured into non-polynomial coefficients problems adopted the use of an alternative method like collocation [5].

In this paper, we have set forth a technique for approximating this type of products in terms of Chebyshev series to a level of precision over a large range of $x$. This will in turn facilitates the application of a number of methods on non-polynomial variable coefficients differential equations.

\section{Chebyshev Polynomials}

For easy reference, the definitions and certain basic properties of the Chebyshev polynomials are presented. The properties are needed in illustrating our main results.

The Chebyshev polynomials of the first kind are in this work denoted by $T_{r}(x)$ and are defined as a set of orthogonal polynomial of degree $r$ given by:

$$
T_{r}(x)=\cos \left\{r \cos ^{-1}\left(\frac{2 x-b-a}{b-a}\right)\right\}
$$

and satisfies the recurrence relation;

$$
T_{r+1}(x)=2\left(\frac{2 x-b-a}{b-a}\right) T_{r}(x)-T_{r-1}(x)
$$

which is valid within the interval $a \leq x \leq b$, (see ref [6] and [7])

The usefulness of this polynomial is enormous especially in the field of numerical approximation for interval $-1 \leq x \leq 1$. The following are the properties exhibited within this interval.

- $\left|T_{r}(x)\right| \leq 1$

- The maxima and minima are of comparable value.

- The maxima and minima are spread reasonably uniformly. 
- All Chebyshev polynomials satisfy a three term recurrence relation.

- They are easy to compute and to convert to and from a power series form.

These properties together produced an approximating polynomial which minimizes errors in its application. According to [6] and [8], this is different from the least squares approximation where the sum of the squares of the errors is minimized; the maximum error itself can be quite large. In Chebyshev approximation, the average error can be large but the maximum error is minimized. For this, Chebyshev approximations of a function are sometimes said to be mini-max approximation [2].

\section{Numerical Techniques}

For a linear problems with linear associated conditions and whose coefficients and terms are themselves polynomials, authors like Clenshaw[8], Fox[3] applied simple formula which enables both sides of the differential equation to be expressed as sum of Chebyshev polynomials, the coefficients on the left being linear combination of the $a_{i}$. Such important formulae include;

which in general gives

$$
x T_{r}(x)=\frac{1}{2}\left(T_{r-1}(x)+T_{r+1}(x)\right)
$$

$$
x^{k} T_{r}(x)=\frac{1}{2^{k}} \sum_{i=0}^{k}\left(\begin{array}{l}
k \\
i
\end{array}\right) T_{r-k+2 i}(x)
$$

For the shifted interval $0 \leq x \leq 1$ (4) becomes

$$
x^{k} T_{r}^{*}(x)=\frac{1}{2^{2 k}} \sum_{i=0}^{2 k}\left(\begin{array}{c}
2 k \\
i
\end{array}\right) T_{r-k+i}^{*}(x)
$$

where $T^{*}(x)$ is Chebyshev polynomials shifted into interval $0 \leq x \leq 1$

Formulas like $\quad T_{m}(x) T_{n}(x)=\frac{1}{2}\left\{T_{m+n}(x)+T_{|m-n|}(x)\right\}$

have equally been established in literatures for problems in numerical approximation, see( ref. [2]).

For nonlinear problems and problems whose coefficients and other terms are nonpolynomials,technique of taylor series representation for each function $f(x)$ at $x=a$ is hereby adopted as;

$f(x)=f(x)+(x-a) f^{\prime}(a)+\frac{(x-a)^{2}}{2 !} f^{\prime \prime}(a)+\cdots+\frac{(x-a)^{n-1}}{(n-1) !} f^{n-1}(a)+R_{n}(x)$

where

$R_{n}(x)=\frac{(x-a)^{n}}{n !} f^{n}(\theta)$

$a \leq \theta \leq x$

Each of these is carried out for varrying degree $n$ of each expansion.

IV. Experiments Of The Technique And Results

In this section, the use of the discussed technique is displayed and numerical experiment on some notable functions is carried out. This is done for varrying degree of $n$ of each expansion. The MATLAB program that does this is written and structured to accept numeric coefficients of $x$ in each expansion of the function $f(x)$.

4.1 Chebyshev Representation For Product $T_{r}(x) e^{x}$

In taylor series form;

$$
\begin{array}{r}
e^{x}=\sum_{k=0}^{\infty} \frac{x^{k}}{k !} \\
\therefore \quad e^{x} T_{r}(x)=\sum_{k=0}^{\infty} \frac{x^{k} T_{r}(x)}{k !}
\end{array}
$$

substituting (4) into (10) and taking its finite sum, yields; 
$T_{r}(x) e^{x}=\sum_{k=0}^{n} \sum_{i=0}^{k} \frac{T_{r-k+2 i}(x)}{2^{k}(k-i) ! i !}$

For $\mathrm{n}=1,2,3,4$ and 5 , we have ;

Table 1: Chebyshev polynomials equivalence of product $T_{r}(x) e^{x}$

\begin{tabular}{|l|l|}
\hline $\mathrm{N}$ & $T_{r}(x) e^{x}$ \\
\hline 1 & $\frac{1}{2}\left(T_{r-1}+2 T_{r}+T_{r+1}\right)$ \\
\hline 2 & $\frac{1}{8}\left(T_{r-2}+4 T_{r-1}+10 T_{r}+4 T_{r+1}+T_{r+2}\right)$ \\
\hline 3 & $\frac{1}{48}\left(T_{r-3}+6 T_{r-2}+27 T_{r-1}+60 T_{r}+27 T_{r+1}+6 T_{r+2}+T_{r+3}\right)$ \\
\hline 4 & $\frac{1}{384}\left(T_{r-4}+8 T_{r-3}+52 T_{r-2}+216 T_{r-1}+480 T_{r}+216 T_{r+1}+52 T_{r+2}+8 T_{r+3}+T_{r+4}\right)$ \\
\hline 5 & $\frac{1}{3840}\left(T_{r-5}+10 T_{r-4}+85 T_{r-3}+520 T_{r-2}+2170 T_{r-1}+4860 T_{r}+2170 T_{r+1}+520 T_{r+2}\right.$ \\
& $\left.+85 T_{r+3}+10 T_{r+4}+T_{r+5}\right)$ \\
\hline
\end{tabular}

*The argument $x$ is omitted for simplicity.

4.2 Chebyshev Series Representation For Product $T_{r}(x) \cos x$

$\cos x=\sum_{k=0}^{\infty} \frac{(-1)^{k} x^{2 k}}{2 k !}$

$T_{r}(x) \cos x=\sum_{k=0}^{\infty} \frac{(-1)^{k} x^{2 k} T_{r}(x)}{2 k !}$

From (4), we have;

$x^{2 k} T_{r}(x)=\frac{1}{2^{2 k}} \sum_{i=0}^{2 k}\left(\begin{array}{c}2 k \\ i\end{array}\right) T_{r-2 k+2 i}(x)$

substituting (14) into (13), we have;

$T_{r}(x) \cos x=\sum_{k=0}^{\lfloor n / 2\rfloor 2 k} \sum_{i=0} \frac{\left(-\frac{1}{4}\right)^{k} T_{r-2 k+2 i}(x)}{(2 k-i) ! i !}$

For $\mathrm{n}=1,2,3,4$ and 5, we have;

Table 2: Chebyshev polynomials equivalence of product $T_{r}(x) \cos x$

\begin{tabular}{|l|l|}
\hline $\mathrm{n}$ & $T_{r}(x) \cos x$ \\
\hline 1 & $T_{r}(x)$ \\
\hline 2 & $\frac{1}{8}\left(-T_{r-2}+6 T_{r}-T_{r+2}\right)$ \\
\hline 3 & $\frac{1}{8}\left(-T_{r-2}+6 T_{r}-T_{r+2}\right)$ \\
\hline 4 & $\frac{1}{384}\left(T_{r-4}-44 T_{r-2}+294 T_{r}-44 T_{r+2}+T_{r+4}\right)$ \\
\hline 5 & $\frac{1}{384}\left(T_{r-4}-44 T_{r-2}+294 T_{r}-44 T_{r+2}+T_{r+4}\right)$ \\
\hline
\end{tabular}

4.3 Chebyshev Series Representation For Product $T_{r}(x) \sin x$

$$
\begin{aligned}
& \sin x=\sum_{k=0}^{\infty} \frac{(-1)^{k} x^{2 k+1}}{(2 k+1) !} \\
& T_{r}(x) \sin x=\sum_{k=0}^{\infty} \frac{(-1)^{k} x^{2 k+1} T_{r}(x)}{(2 k+1) !}
\end{aligned}
$$


From (4), we have;

$x^{2 k+1} T_{r}(x)=\frac{1}{2^{2 k+1}} \sum_{i=0}^{2 k+1}\left(\begin{array}{c}2 k+1 \\ i\end{array}\right) T_{r-2 k+2 i-1}(x)$

from (18), we have the finite form of (17), we have;

$T_{r}(x) \sin x=\frac{1}{2} \sum_{k=0}^{\left\lfloor\frac{n-1}{2}\right\rfloor} \sum_{i=0}^{2 k+1} \frac{\left(-\frac{1}{4}\right)^{k} T_{r-2 k+2 i-1}(x)}{(2 k-i+1) ! i !}$

For $n=1,2,3,4$ and 5, we have;

Table 3: Chebyshev polynomials equivalence of $T_{r}(x) \sin x$

\begin{tabular}{|l|l|}
\hline $\mathrm{n}$ & $T_{r}(x) \sin x$ \\
\hline 1 & $\frac{1}{2}\left(T_{r-1}+T_{r+1}\right)$ \\
\hline 2 & $\frac{1}{2}\left(T_{r-1}+T_{r+1}\right)$ \\
\hline 3 & $\frac{1}{48}\left(-T_{r-3}+21 T_{r-1}+21 T_{r+1}-T_{r+3}\right)$ \\
\hline 4 & $\frac{1}{48}\left(-T_{r-3}+21 T_{r-1}+21 T_{r+1}-T_{r+3}\right)$ \\
\hline 5 & $\frac{1}{3840}\left(T_{r-5}+75 T_{r-3}+1690 T_{r-1}+1690 T_{r+1}-75 T_{r+3}+T_{r+5}\right)$ \\
\hline
\end{tabular}

4.4Chebyshev Series Representation For Product $T_{r}(x) \tan x$

$\tan x=\sum_{k=0}^{\infty} \frac{A_{2 k+1} x^{2 k+1}}{(2 k+1) !}$

Where $A_{2 k+1}$ are the tangent number.

$T_{r}(x) \tan x=\sum_{k=0}^{\infty} \frac{A_{2 k+1} x^{2 k+1} T_{r}(x)}{(2 k+1) !}$

Substituting (18) into (21) and taking the finite sums we have;

$T_{r}(x) \tan x=\sum_{k=0}^{\left\lfloor\frac{n-1}{2}\right.} \sum_{i=0}^{2 k+1} \frac{A_{2 k+1} T_{r-2 k+2 i-1}(x)}{2^{2 k+1}(2 k-i+1) ! i !}$

For $\mathrm{n}=1,2,3,4$ and 5, we have;

Table 4: Chebyshev polynomials equivalence of product $T_{r}(x) \tan x$

\begin{tabular}{|l|l|}
\hline $\mathrm{N}$ & $T_{r}(x) \tan x$ \\
\hline 1 & $\frac{1}{2}\left(T_{r-1}+T_{r+1}\right)$ \\
\hline 2 & $\frac{1}{2}\left(T_{r-1}+T_{r+1}\right)$ \\
\hline 3 & $\frac{1}{24}\left(T_{r-3}+15 T_{r-1}+15 T_{r+1}+T_{r+3}\right)$ \\
\hline 4 & $\frac{1}{24}\left(T_{r-3}+15 T_{r-1}+15 T_{r+1}+T_{r+3}\right)$ \\
\hline 5 & $\frac{1}{240}\left(T_{r-5}+15 T_{r-3}+160 T_{r-1}+160 T_{r+1}+15 T_{r+3}+T_{r+5}\right)$ \\
\hline
\end{tabular}

For hyperbolic functions, we have;

$$
\begin{aligned}
& T_{r}(x) \cosh x=\sum_{k=0}^{\left\lfloor\frac{n}{2}\right\rfloor} \sum_{i=0}^{2 k} \frac{T_{r-2 k+2 i-1}(x)}{4^{k}(2 k-i) ! i !} \\
& T_{r}(x) \sinh x=\sum_{k=0}^{\left\lfloor\frac{n-1}{2}\right\rfloor_{2=0}^{2 k+1}} \frac{T_{r-2 k+2 i-1}(x)}{2^{2 k+1}(2 k+1-1) ! i !}
\end{aligned}
$$


$T_{r}(x) \tanh x=\sum_{k=0}^{\left\lfloor\frac{n-1}{2} \sum_{i=0}^{2 k+1} \frac{(-1)^{k} A_{2 k+1} T_{r-2 k+2 i-1}(x)}{2^{2 k+1}(2 k+1-i) ! i !}\right.}$

All these are valid for the range $-1 \leq x \leq 1$.

For range $0 \leq x \leq 1$, the following derivations are established.

$T_{r}^{*}(x) e^{x}=\sum_{k=0}^{n} \sum_{i=0}^{2 k} \frac{\left(\begin{array}{c}2 k \\ i\end{array}\right) T_{r-k+i}^{*}(x)}{2^{2 k} k !}$

$T_{r}^{*}(x) \cos x=\sum_{k=0}^{\lfloor n / 2\rfloor} \sum_{i=0}^{4 k} \frac{\left(\begin{array}{c}4 k \\ i\end{array}\right) T_{r-2 k+i}^{*}(x)}{(-2)^{4 k} 2 k !}$

$T_{r}^{*}(x) \sin x=\frac{1}{4} \sum_{k=0}^{\left\lfloor\frac{n-1}{2}\right.} \sum_{i=0}^{4 k+2} \frac{\left(\begin{array}{c}4 k+2 \\ i\end{array}\right) T_{r-2 k+2 i-1}^{*}(x)}{(-2)^{4 k}(2 k+1) !}$

$T_{r}^{*}(x) \tan x=\sum_{k=0}^{\left\lfloor\frac{n-1}{2}\right\rfloor} \sum_{i=0}^{4 k+2} \frac{A_{2 k+1}\left(\begin{array}{c}4 k+2 \\ i\end{array}\right) T_{r-2 k+2 i-1}^{*}(x)}{2^{4 k+2}(2 k+1) !}$

$T_{r}^{*}(x) \cosh x=\sum_{k=0}^{\lfloor n / 2\rfloor} \sum_{i=0}^{4 k} \frac{\left(\begin{array}{c}4 k \\ i\end{array}\right) T_{r-2 k+i}^{*}(x)}{2^{4 k}(2 k) !}$

$T_{r}^{*}(x) \sinh x=\sum_{k=0}^{\left\lfloor\frac{n-1}{2}\right\rfloor} \sum_{i=0}^{4 k+2} \frac{\left(\begin{array}{c}4 k+2 \\ i\end{array}\right) T_{r-2 k+i-1}^{*}(x)}{2^{4 k+2}(2 k+1) !}$

$T_{r}^{*}(x) \tanh x=\sum_{k=0}^{\left\lfloor\frac{n-1}{2}\right.} \sum_{i=0}^{4 k+2} \frac{(-1)^{k} A_{2 k+1} T_{r-2 k+2 i-1}^{*}(x)}{2^{4 k+2}(2 k+1) !}$

*where $k$ and $i$ are integer.

\section{Conclusion}

The technique that makes expansion method applicable to problems with non-polynomial terms has been studied. The analyzed techniques in conjunction with other properties of Chebyshev polynomials listed in section 3 makes feasible the application of expansion method to a vase class of problems.

On this note, we conclude that expansion method has been extended to cover a wider range of problems covering nonlinear and non-polynomial coefficients differential equations.

\section{References}

Grewal,B.S.Numerical methods in Engineering and science, 7th ed. Kanna Publishers Delhi, 2005.

Fox, L. and Parker,I. B.Chebyshev Polynomials in Numerical analysis, Oxford University press NY Toroto, 1968.

Fox, L.The use and construction of Mathematical tables, Math. Tab. Phys. Lab. 1, London, H. M. Stat. office, 1956.

Mason,J. C. Some new approximations for the solution of Differential Equations, D. Phil., Oxford Univeristy. 1965

Aysegul, A.,Chebyshev Polynomials in Numerical approximations for PDEs with complicated condition,Num. Methods for PDEs 25(3), 2008, 610-621.

[6] Mason, J.C. and Handscomb, D.C.,Chebyshev Polynomials, Rhapman \& Hall - CRC, Roca Raton, London, New York, Washington D.C. 2003

[7] Lanczos, C.Legendre Versus Chebyshev polynomials. Miller topics in Numerial analysis, Academic press, London, 1973.

[8] Clenshaw, C. W. A note on the summation of Chebyshev series. Math. Tab Wash. 9, 1955, 119-120 